\title{
Comprehensive Morphological Assessment of Cord Blood: Normal Values and the Prevalence of Morphologically Aberrant Leukocytes
}

\author{
Ben-Zion Katz ${ }^{a} b$ Dan Benisty ${ }^{a}$ Sigi Kay ${ }^{a}$ Jacky Herzlich ${ }^{c}$ Craig Raskind $^{c}$ \\ Ronella Marom ${ }^{b, c}$ \\ aDivision of Hematology, Tel Aviv Sourasky Medical Center, Tel Aviv, Israel; bSackler Faculty of Medicine, Tel Aviv \\ University, Tel Aviv, Israel; 'Department of Neonatology, Dana Dwek Children Hospital, Tel Aviv Sourasky Medical \\ Center, Tel Aviv, Israel
}

\section{Keywords}

Aberrant lymphocytes · Cord blood · Morphology · Preterm newborn

\begin{abstract}
Introduction: Cord blood (CB) is becoming a valuable source for stem cells utilized in a variety of cell therapy applications, as well as for newborn diagnostics. Some parameters of the $\mathrm{CB}$ cellular components can be provided by automated analyzers, while others, such as immature or aberrant cells, require blood film morphological assessment. The objectives of the study were to establish normal CB morphology and to determine the prevalence of morphologically aberrant leukocytes in CB. Methods: We performed a comprehensive morphological analysis of 100 CB samples taken from healthy term and appropriate-for-gestational-age neonates born to healthy mothers, preterm neonates, neonates of diabetic mothers, and small-for-gestational-age neonates. Blood counts were assessed, and manual morphological analyses were performed by laboratory specialists. Results: The manual differential count of normal CB samples established the following values: $47.8 \pm 10.7 \%$ neutrophils, $31.2 \pm 9.8 \%$ lymphocytes, $10.0 \pm 4.0 \%$ monocytes, and $3.0 \pm 2.5 \%$ eosino-
\end{abstract}

karger@karger.com www.karger.com/aha

Karger $\stackrel{\text { ' }}{5}$

GOPEN ACCESS
(C) 2021 The Author(s)

Published by S. Karger AG, Basel

This is an Open Access article licensed under the Creative Commons Attribution-NonCommercial-4.0 International License (CC BY-NC) (http://www.karger.com/Services/OpenAccessLicense), applicable to the online version of the article only. Usage and distribution for commercial purposes requires written permission. phils, with no significant sex-related differences. Blasts were observed in 44/100 samples with an average of $0.5 \pm 0.7 \%$ per sample, and only a minor left shift was observed. There were significant populations of large granular lymphocytes $(19.1 \pm 10.6 \%$ of the total lymphocytes) and morphologically aberrant lymphocytes $(12.4 \pm 5.4 \%$ of the total lymphocytes) in the samples, irrespective of neonatal status. The differentials of preterm CB samples differ significantly from normal term $\mathrm{CB}$ samples, including the reverse of neutrophils/lymphocytes ratio, and the lack of basophils. Conclusions: Normal values and unique morphological features in the $C B$ of neonates are described. The abundant morphologically aberrant lymphocytes in CB may represent an immature state of the immune system at birth. @ 2021 The Author(s).

Published by S. Karger AG, Basel

\section{Introduction}

For several decades, cord blood (CB) has been a valuable source of hematopoietic stem cells both for research and for various clinical applications [1,2]. New potentials are continuously emerging in the field of $\mathrm{CB}$-derived therapy, including gene editing [1] and tissue regenera- 
tion [3]. However, CB can also be utilized for the diagnosis of various diseases and conditions in the newborn. Several attempts have been made to sample CB for diagnostic purposes. For example, it has been suggested that $\mathrm{CB}$ could be tested for bilirubin, direct antiglobulin, and ABO typing in order to detect significant hyperbilirubinemia in neonates [4]. Some laboratory tests performed on $\mathrm{CB}$, including the measurement of hepcidin [5], interleukin (IL)-6, and procalcitonin [6], can be used as biomarkers for early-onset neonatal sepsis. Several studies have indicated that $\mathrm{CB}$-derived complete blood count $(\mathrm{CBC})$ can often spare the need to draw blood samples from newborns, which is especially problematic for premature infants [7-9]. Hematological reference ranges for CB have been established [10], but CBC-derived differential counts may not be dependable [11]. While CB-derived $\mathrm{CBCs}$ may provide information on critical clinical parameters, such as hemoglobin levels and platelets counts, they do not provide information on other parameters, such as the levels of reactive lymphocytes, immature cells, and blasts. Those latter parameters can be important for diagnosing clinical entities, such as sepsis, the neonatal syndrome transient abnormal myelopoiesis, and the subsequent Down syndrome-associated acute myeloid leukemia [12], but they can only be obtained from morphological assessments. Previous studies indicated that leukocyte populations in the CB may differ from normal peripheral blood (PB), not only with respect to the presence of immature cells, but other cell types as well. For example, natural killer (NK) cells constitute up to $10 \%$ of lymphocytes in $\mathrm{PB}$ and up to $30 \%$ in $\mathrm{CB}$ [13]. The aim of this study was to determine normal CB morphology and to identify morphological abnormalities in $\mathrm{CB}$.

\section{Materials and Methods}

\section{Sample Collection}

One hundred samples were collected over a period of a year. The 100 analyzed CB samples were from 54 male and 46 female neonates. The umbilical CB samples were collected ex utero after clamping of the cord prior to expulsion of the placenta. CB collections were carried out by obstetricians or midwives in the delivery room. The samples were collected into a spray-dried $\mathrm{K}_{3}$ EDTA vacuum tube (Greiner, Kremsmunster, Austria), and were analyzed in DxH800 Beckman Coulter CBC analyzers (Brea, CA, USA).

\section{Clinical Parameters}

Clinical parameters were retrospectively reviewed from the medical records and were evaluated for their potential effect on $\mathrm{CB}$ values. Those parameters included gestational age (GA), sex, birth weight, APGAR score, and clinical information on the maternal health status, such as chronic diseases, abnormalities and/or complications of labor, and prior and current drug treatment. GA was determined by the first-trimester ultrasound, by obstetrical dating of the pregnancy, and/or by examination of the infant after birth.

\section{Blood Film Preparation and Morphological Analysis}

The average period from sample collection to CBC count/slide preparation in the laboratory is $26 \pm 41 \mathrm{~min}$. Blood films were automatically prepared on glass slides (SP-Slides, Sysmex, Lincolnshire, IL, USA) by a Beckman Coulter Slide Maker Stainer (Brea, CA, USA) and stained according to the following protocol: methanol (absolute, Bio-Lab Ltd., Jerusalem, Israel) 4 min $1 \mathrm{~s}$; May Grunwald stain (TruColor, Beckman Coulter, Brea, CA, USA) 6 min $22 \mathrm{~s}$; water (double distilled, Terion, Cary, NC, USA) 3 min; Giemsa stain (TruColor, Beckman Coulter, Brea, CA, USA) 9 min; water (double distilled, Terion, Cary, NC) 1 min; air dry: 6 min. Morphological analysis was performed by the DM1200 CellaVision image analyzer, followed by review and approval by a certified laboratory specialist. A total of 150 cells were collected for each analysis. Detailed analysis of lymphocytes morphology by a certified laboratory specialist was performed on 100 lymphocytes (100\% total). Aberrations in lymphoid cells in the PB were defined by the ICSH as "... increased cell size, immaturity of the nucleus including a visible nucleolus and lack of chromatin condensation, irregular nuclear outline or lobulation, cytoplasmic basophilia and vacuolation, and irregular cell outline. The cytoplasm may be abundant with staining varying from pale blue to markedly basophilic especially at points of contact with adjacent cells" [14]. Reactive lymphocytes were classified as cells that characterize viral infections, and include "variant, reactive, abnormal, activated and atypical lymphocytes, Downey cells Type 1-3, Turk cells, immunoblasts and even combinations of cells," according to the definition by the ICSH [14].

\section{Statistical Analysis}

Statistical significances of the experiments presented in Figures $1-4$, as well as in online supplementary Figure 1 (for all online suppl. material, see www.karger.com/doi/10.1159/000520638) were determined by Student's $t$ test, and $p$ values $<0.05$ were considered statistically significant.

\section{Results}

$\mathrm{CBC}$ data of the samples were evaluated prior to the morphological analysis. As shown in Figure 1a, CBC-derived parameters pointed to significantly lower hemoglobin levels in the female CB samples (mean \pm standard deviation $15.01 \pm 1.46 \mathrm{~g} / \mathrm{dL}$ ) than the male CB samples $(15.7 \pm 1.66 \mathrm{~g} / \mathrm{dL})$. The lower hemoglobin levels in the female samples stemmed from lower red blood cell (RBC) numbers, rather than from differences in the mean hemoglobin concentrations (Fig. 1a). These differences are specific to the RBC lineage, and not to other blood components, such as white blood cells (WBCs) and platelets that were similar for the male and female samples (Fig. 1a). 

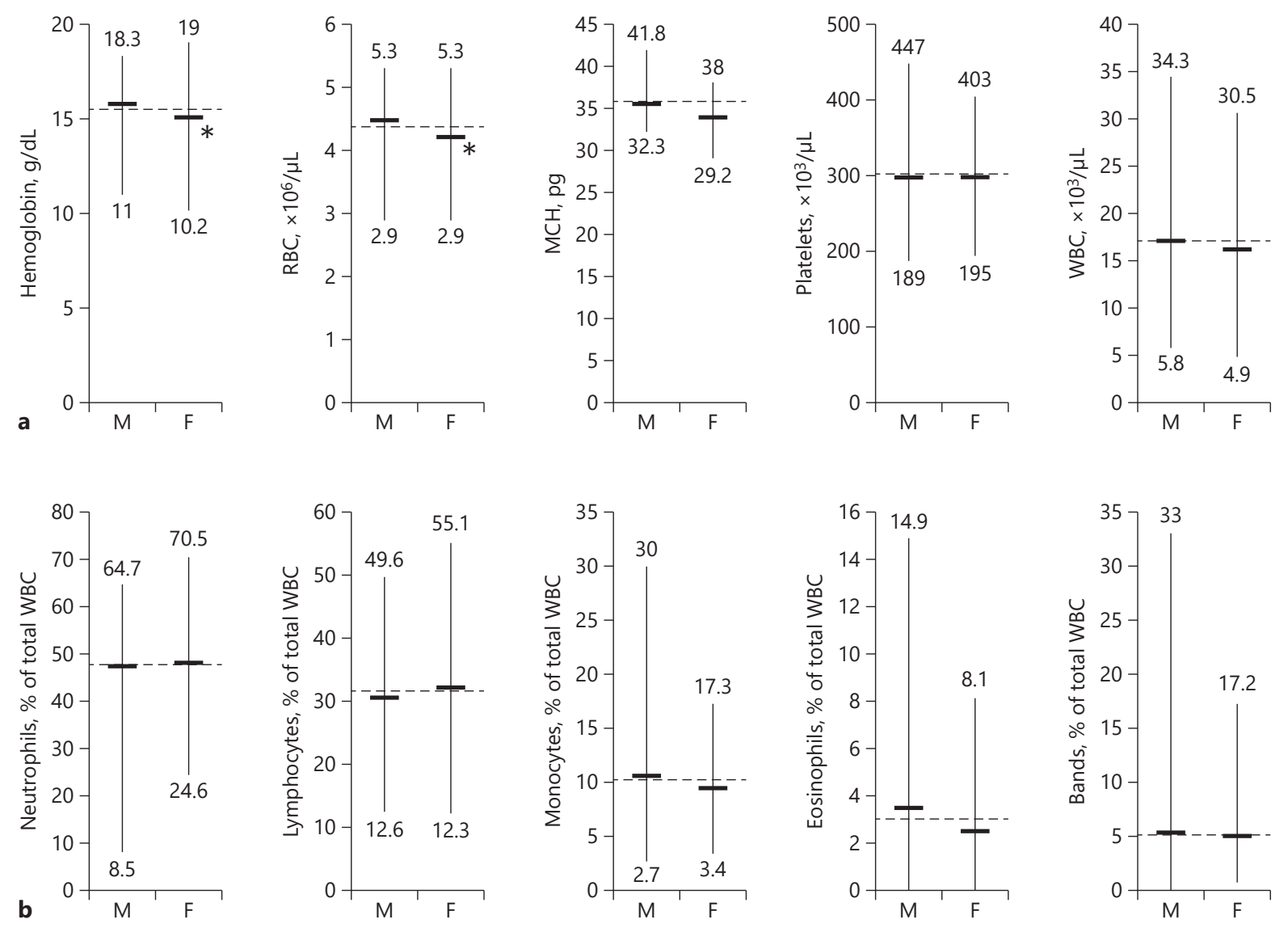

Fig. 1. a Hematological assessment of $C B$ samples. $C B C$ was performed on $100 \mathrm{CB}$ samples. Distribution of hemoglobin, RBC, $\mathrm{MCH}$, platelets, and WBC are presented according to sex variable (F - female, $N=46 ; \mathrm{M}$ - male, $N=54$ ). High, low, and average values (detailed in the text) for each parameter are shown. The overall average (dashed line) is shown with the averages and distribution among female $(\mathrm{F})$ and male $(\mathrm{M})$ samples. ${ }^{*}$ Significant $(p$ $<0.007)$ difference between female and male samples. b Morpho- logical assessment of CB samples. Manual differential count was performed on $100 \mathrm{CB}$ samples. Differential counts of the major types of leukocytes according to sex variable ( $\mathrm{F}-$ female, $N=46$; $\mathrm{M}$ - male, $N=54$ ) are presented. The overall average (dashed line) is shown with the averages and distribution of samples among female $(\mathrm{F})$ and male $(\mathrm{M})$ samples. $\mathrm{CB}$, cord blood; $\mathrm{CBC}$, complete blood count; RBCs, red blood cells; $\mathrm{MCH}$, mean corpuscular hemoglobin; WBC, white blood cells.
The manual differential count established the following values: $47.8 \pm 10.7 \%$ neutrophils, $31.2 \pm 9.8 \%$ lymphocytes, $10.0 \pm 4.0 \%$ monocytes, and $3.0 \pm 2.5 \%$ eosinophils, with no significant sex-related differences (Fig. 1b). As expected, low basophils levels were detected in the $\mathrm{CB}$ samples $(0.5 \pm 0.7 \%)$, with no sex-related differences for them either (data not shown). Analysis of left shift parameters (indicating an increase in the number of immature myeloid cells) revealed an average of $5.1 \pm 4.1$ bands, again with no sex-related difference (Fig. 1b). Only $7 / 100$ samples had $>10 \%$ bands, and one of them had 33\% bands. Excluding the single exceptional sample, the mean band level was similar to the overall value $(4.8 \pm 3.0 \%)$. Other immature myeloid forms included the averages of $0.9 \pm 0.9 \%$ metamyelocytes, $0.5 \pm 0.7 \%$ myelocytes, and $0.6 \pm 0.9 \%$ promyelocytes. Blasts were observed in $44 / 100$ samples, with a mean of $0.5 \pm 0.7 \%$ per sample. No morphological aberrations were observed in the myeloid cells. A detailed analysis of lymphocytes was carried out with the aim of evaluating the prevalence of informative forms, including normal lymphocytes, large granular lymphocytes (LGLs) (Fig. 2a), reactive lymphocytes (Fig. 2b), and aberrant lymphocytes (Fig. 2c). Some of the aberrant cells displayed immature nucleus, including blast-like 

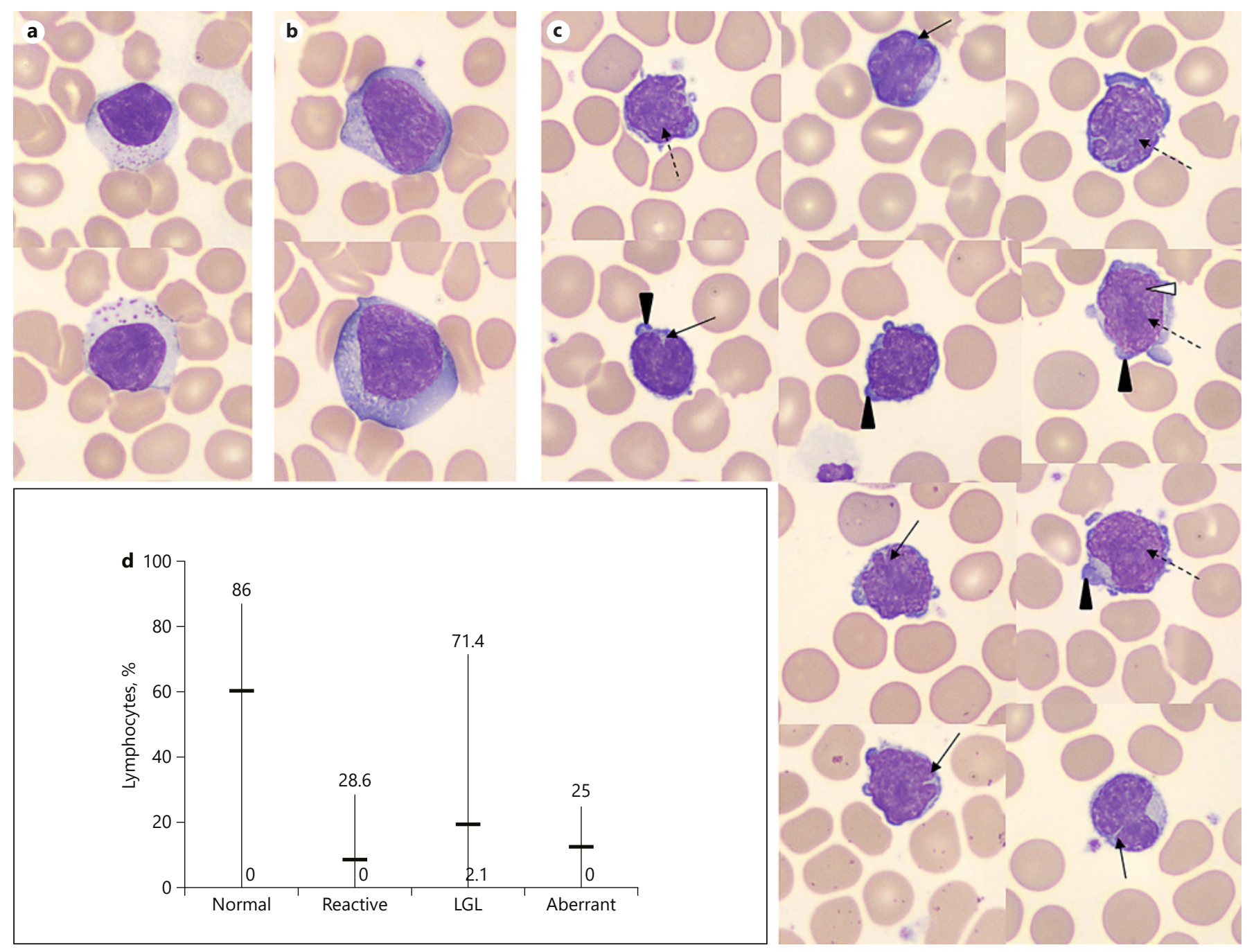

Fig. 2. Morphological assessment of lymphocytes subtypes. Representative examples of: LGLs (a), Reactive (viral) lymphocytes (b), Aberrant lymphocytes (c). Arrows: cleft nucleus, black arrowheads: cytoplasmic blebs, and dashed arrows: reticulated nucleus and white arrowhead: nucleoli (blast-like cell). $\mathbf{d}$ Differential count of lymphocytes subtypes. High, low, and average values (detailed in the text) for each parameter are shown.LGL, large granular lymphocytes; SD, standard deviation. nucleus, with nucleoli (Fig. 2c). The analysis revealed relatively high levels of reactive lymphocytes $(8.3 \pm 6.1 \%$ of the total lymphocytes), LGLs $(19.1 \pm 10.6 \%$ of the total lymphocytes), and aberrant lymphocytes (12.4 $\pm 5.4 \%$ of the total lymphocytes) (Fig. 2d). Aberrant lymphoid features that were observed included cells with cleft nuclei, nucleoli, split nuclei, reticulated nucleus, and irregular membranes (Fig. 2c).

Twelve of the $100 \mathrm{CB}$ samples were from neonates born to mothers with hypothyroidism $(n=3)$, post term delivery $(n=3)$, cytomegalovirus infection during pregnancy $(n=1)$, selective serotonin reuptake inhibitors treatment by the mother $(n=1)$, antibiotic treatment dur- ing labor $(n=1)$ - all with small numbers $(<5)$, and 3 that were not available for further analysis. Therefore, these 12 samples were excluded from further analysis. The remaining 88 samples included a group of 31 samples of neonates who were born to healthy mothers and were appropriate-for-GA, born at term, and had an APGAR score above 8 in $1 \mathrm{~min}$ (defined as normal). There were 14 samples of $\mathrm{CB}$ with a maternal diabetic background, 8 from small-for-gestational-age newborns, 20 from largefor-gestational-age newborns, 10 from preterm newborns, and 5 from newborns with low APGAR scores (Fig. 3). By definition, the cohort of preterm infants was defined by GA $\leq 37$ (week $35 \pm 3$ ) and was also character- 


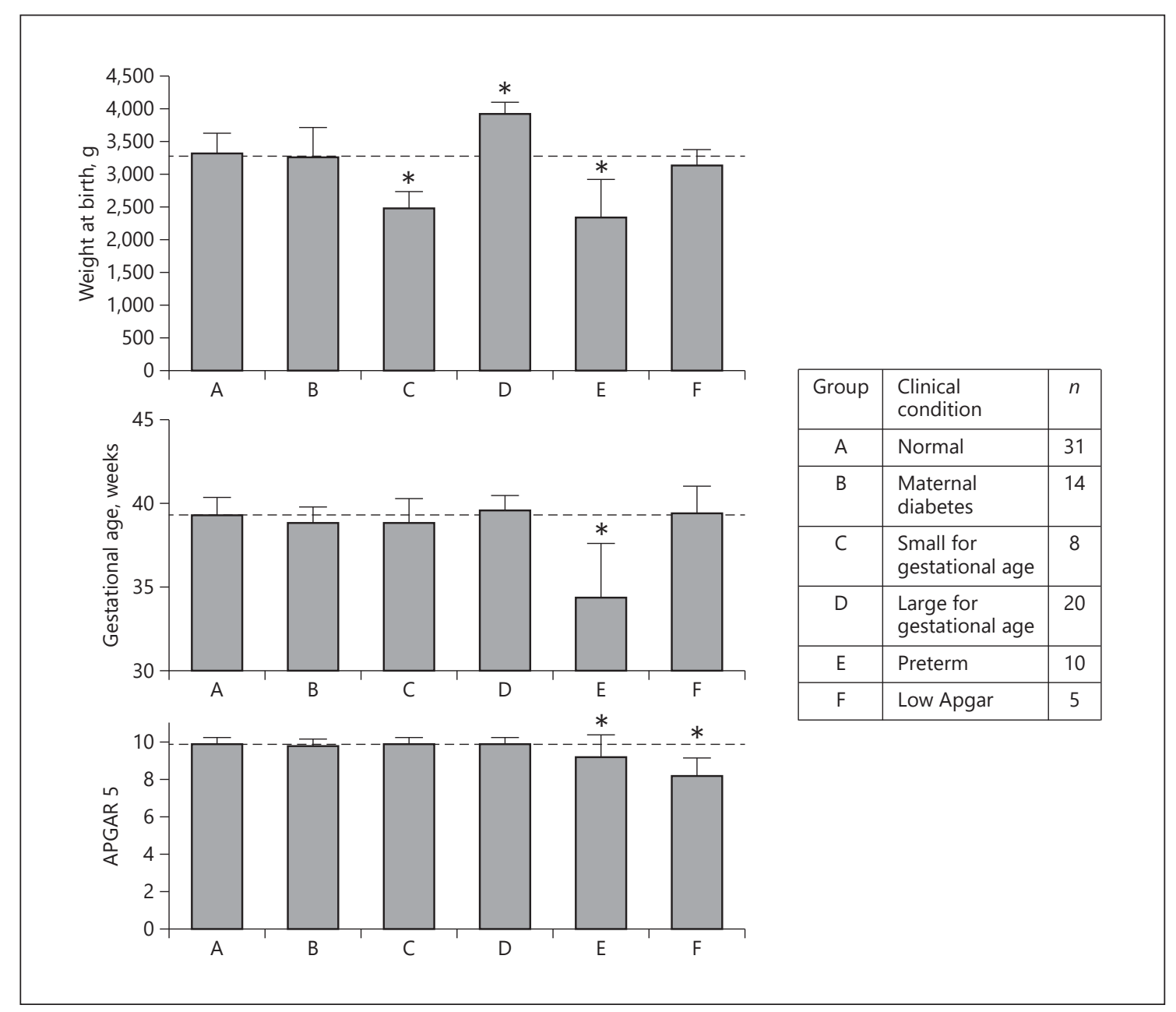

Fig. 3. $\mathrm{CB}$ samples classification according to neonatal status. Eighty-eight $\mathrm{CB}$ samples were analyzed in groups $(\mathrm{A}-\mathrm{F})$ according to different neonatal statuses as depicted in the table (right). Left: the average \pm SD of the weight at birth, GA and APGAR 5 of the

ized by low weight, and small but significant lower APGAR 5 min scores (Fig. 3).

Analysis of CB samples according to neonatal status revealed no differences in hemoglobin and $\mathrm{RBC}$ levels (online suppl. Fig. 1). However, specific low WBC and platelets counts were observed in the preterm group, with some increase in the WBC counts in the low APGAR 5 min score group (online suppl. Fig. 1). While the differential counts were similar in most of the groups (with the exception of a relatively high neutrophil percentage in the maternal diabetic group), the preterm infants samples were noticeably different from the others (Fig. 4). Unlike the other samples which had relatively higher percentages of neutrophils and relatively lower percentages of lymphocytes, this ratio was re- samples in each group. *Significant $(p<0.05)$ difference compared to the normal samples (group A). Dashed line: Group A level. GA, gestational age; LGLs, large granular lymphocytes, SD, standard deviation.

versed in the preterm group (Fig 4). These differences were not accompanied by changes in the left shift, and the proportions of immature neutrophils were similar for all clinical groups (data not shown). In addition, the eosinophil levels were higher in the preterm group (Fig. 4), but there was a single sample with $7.6 \%$ eosinophils which elevated the group mean that would otherwise be similar to that of the other groups (data not shown).

All samples contained low basophil counts, but it reached a level of significance only in the preterm group (Fig. 4), where 6/7 samples had no basophils whatsoever. Similar levels of reactive and aberrant lymphocytes were observed in all groups, with a small increase in the LGL neonates of the maternal diabetic group, the large-for- 

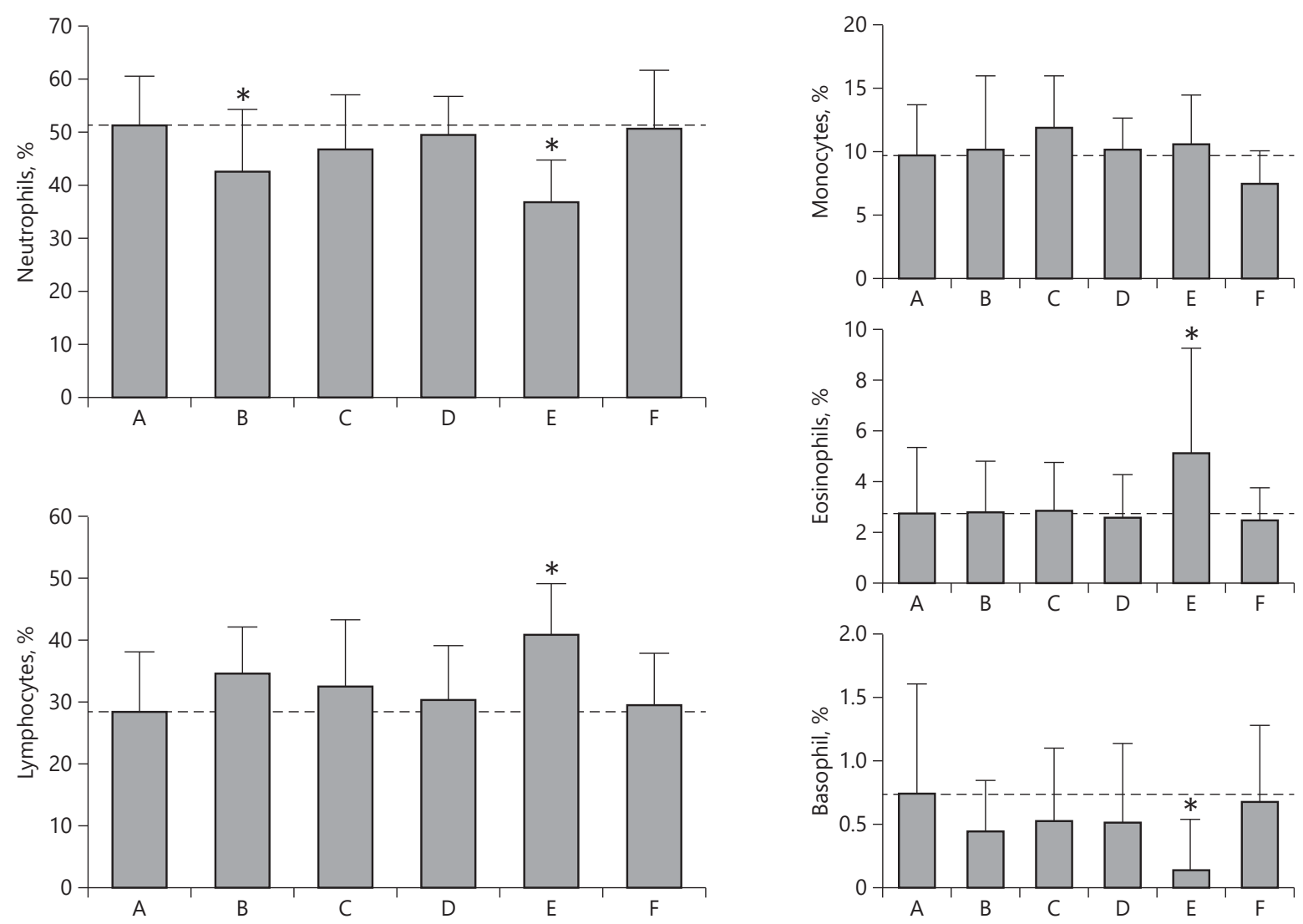

Fig. 4. Differential count of the major types of leukocytes according to neonatal status. The averages \pm SD of $\%$ of neutrophils, lymphocytes, monocytes, eosinophils, and basophils according to different neonatal statuses $(\mathrm{A}-\mathrm{F})$ are shown. ${ }^{*}$ Significant $(p<0.05)$ difference compared to the normal samples (group A). The number of samples are indicated in the table in Figure 3. Dashed line: group A level. gestational-age group, and the low APGAR 5 min score group (Fig. 5).

Nucleated red blood cells (NRBC) were detected in $92 / 100$ samples. Most samples contained 5-10 NRBC/100 WBC, with a mean of $5.7 \pm 6.2 \mathrm{NRBC} / 100 \mathrm{WBC}$ (Fig. 6). $96 / 100$ samples contained up to $20 \mathrm{NRBC} / 100 \mathrm{WBC}$. The samples with $>15 \mathrm{NRBC} / 100 \mathrm{WBC}$ included 2 among the samples of the normal group, 2 in the small-for-gestational-age group, 1 in the preterm group, and 1 in the maternal diabetic group. The morphological assessment of the $\mathrm{RBC}$ pointed to large $\mathrm{RBC}$ as the main characteristic of all samples, compatible with high MCV values of these samples (average 109.4 \pm 4.3 , range 97.7-123.1 fL). Also, relatively high prevalence of small $\mathrm{RBC}$ and polychromasia were observed, and the prevalence of echinocytes to a lesser extent (Fig. 6).

Normal Values and Prevalence of Aberrant Leukocytes in Cord Blood

\section{Discussion}

This study depicts the morphological abnormalities and the characteristics of normal CB in neonates. The current guidelines for the review of blood films do not include criteria for $\mathrm{CB}[14,15]$, and while some reference ranges for $\mathrm{CBC}$-derived parameters in neonatal $\mathrm{CB}$ samples have been documented in the past [10], no comparable determination exists for morphological parameters. Our data indicate that the CBC-derived parameters of our CB cohort reflect previously published data [10]. The most notable RBC morphological feature found in $\mathrm{CB}$ samples was the presence of large $\mathrm{RBC}$, compatible with high MCV values of these samples. Large RBC are considered as normal for newborn RBC population $[10,14]$.

The morphological assessment of the leukocytes in our CB samples pointed to a limited left shift, with single 


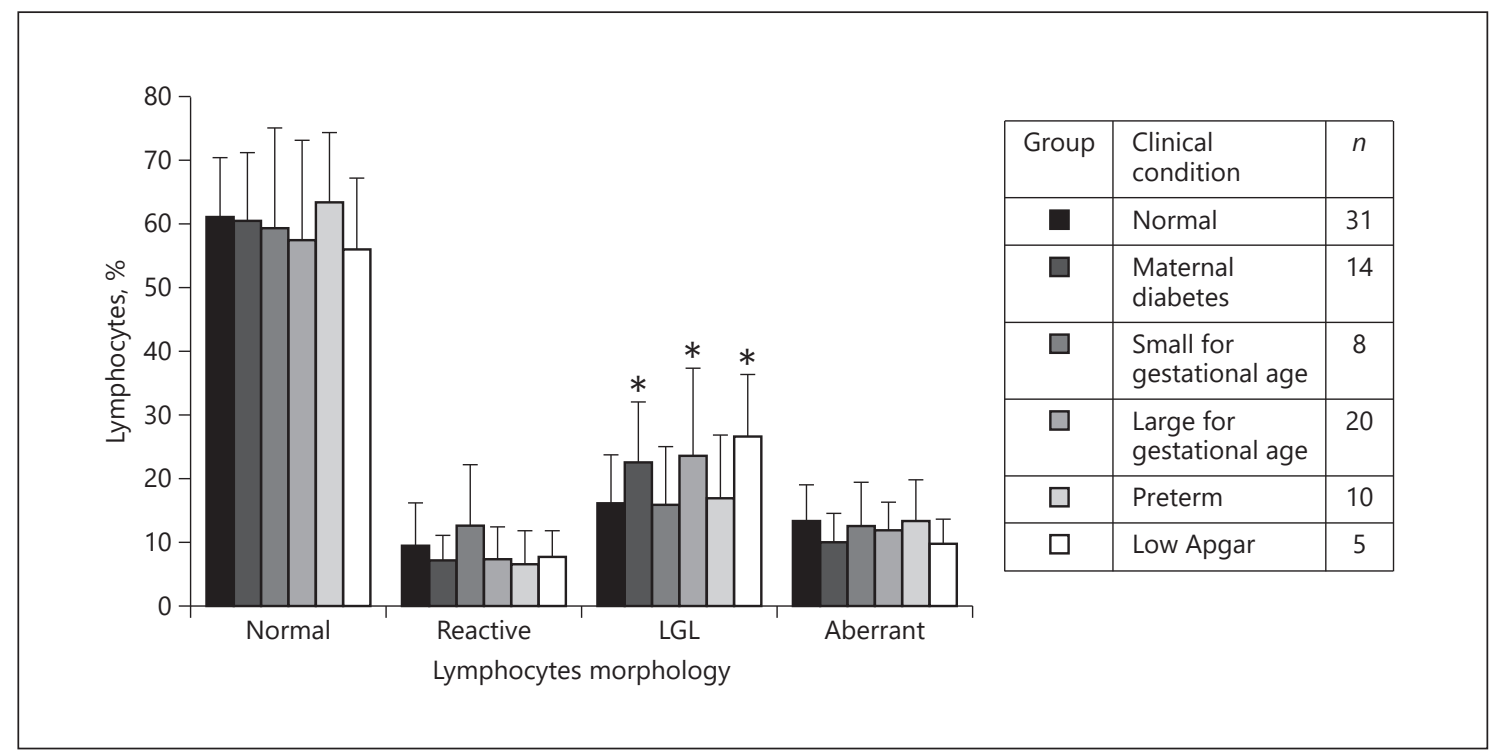

Fig. 5. Differential counts of lymphocytes subtypes according to neonatal status. The column patterns and samples numbers according to neonatal statuses are depicted in the table (right). The averages \pm SD of $\%$ (of total lymphocytes) of normal lymphocytes, reactive lymphocytes LGL and aberrant lymphocytes, according to different neonatal statuses $(\mathrm{A}-\mathrm{F})$ are shown. ${ }^{*}$ Significant $(p<0.05)$ difference compared to the normal samples (group A). GA, gestational age; LGL, large granular lymphocytes; SD, standard deviation.
Fig. 6. RBCs morphological analysis of cord blood samples. The predominant RBC morphological features (out of 100 samples) are depicted in the table (right). Samples were scored positive only if at least $5-10 \%$ of the RBC in the sample had the designated morphology. Left: number of NRBC/100 WBC in cord blood samples. Samples were divided into groups according to their NRBC content. Arrow - the mean NRBC/100 WBC per cord blood sample $(5.7 \pm 6.2)$. RBCs, red blood cells; NRBC, nucleated red blood cells; WBCs, white blood cells.

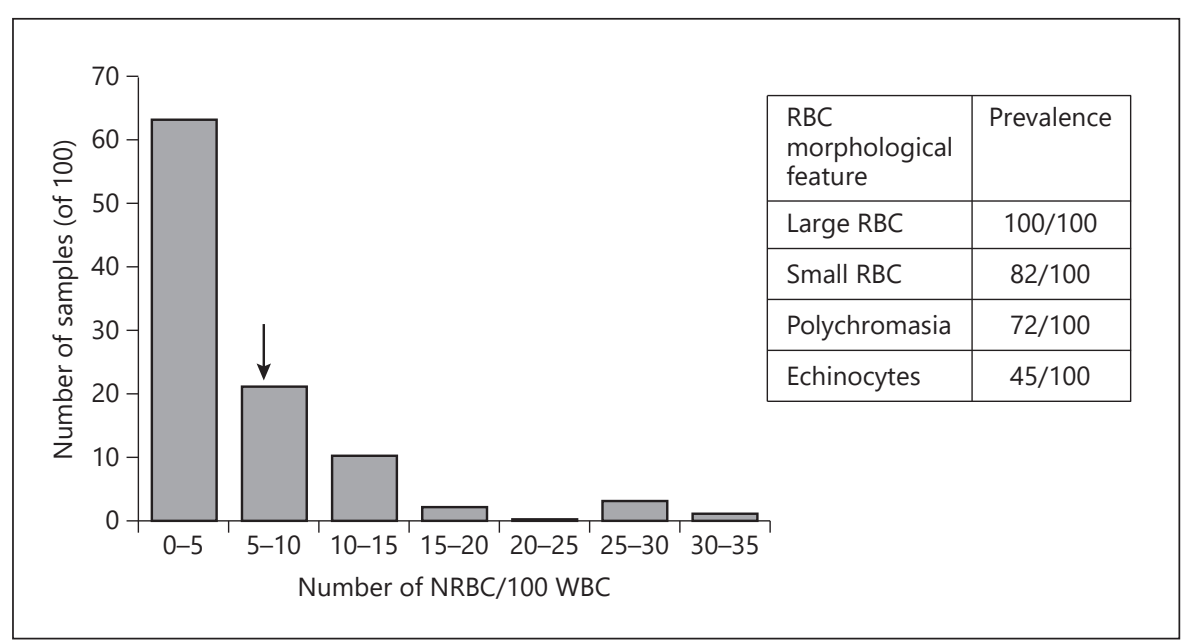

blasts observed in about one-half of the samples. No morphological aberrations were observed in the myeloid cells. In contrast, transient abnormal myelopoiesis has a significantly higher prevalence of both blasts and immature granulocytes (sometimes with aberrant morphology) [16]. The differentials of preterm CB samples differed significantly from those of normal term samples, with lower neutrophils, high lymphocytes, and lower basophils percentages. In a previous study, CBC performed on preterm $\mathrm{CB}$ samples indicated low neutrophil counts in preterm compared to term CB samples, with no difference in lymphocyte counts between preterm and term $\mathrm{CB}$ samples [17].

A significant finding of the current study is the substantial presence of morphologically aberrant (or abnormal) lymphocytes in CB samples, which comprise an average of $12.4 \%$ and up to $25 \%$ of the lymphocytes population in CB samples. These cells were pre-classified by the CellaVision 1200 system as lymphocytes, but contained the following aberrations: increased cell size, immaturity 
of the nucleus, irregular nuclear outline or lobulation, cytoplasmic basophilia, and irregular cell outline. Hence, these cells were classified as aberrant lymphocytes according to previously published definition [14]. As we showed herein, such aberrations were abundant in the $\mathrm{CB}$, and their presence was not associated with disease or specific neonatal conditions. In fact, morphologically aberrant lymphocytes were detected in all but one of the $\mathrm{CB}$ samples, and nearly $60 \%$ of the samples had $>10 \%$ of morphologically aberrant lymphocytes in their lymphocyte populations.

Previous reports noted that incubation of $\mathrm{PB}$ samples in EDTA for prolonged periods ( $>30 \mathrm{~min}$ ) may alter leukocytes morphology, and hence lymphocytes morphology may be affected as well $[18,19]$. However, the average period in our institute from sample collection to $\mathrm{CBC}$ count and slide preparation is $<30 \mathrm{~min}$. Moreover, we did not detect any other morphological aberration in the other cell lineages in this study, and aberrant lymphocytes are not usually observed in normal PB samples processed in the laboratory in identical manner to the CB samples. This indicates that the high prevalence of aberrant lymphocytes do reflect the actual morphological appearance of lymphocytes in $\mathrm{CB}$ samples. In adults, aberrant (or abnormal) lymphocytes should be reported if they appear in significant numbers [14]. However, due to their high prevalence in $\mathrm{CB}$ normal samples, their presence in such samples may not be considered as abnormal.

Reactive lymphocytes typical for viral infections in adults were found in abundance in CB samples. We also observed an average of $19.1 \%$, and up to $71.4 \%$ LGLs of the lymphocyte population in CB samples. Albeit LGL is not a definition of cell population characterized by flow cytometric analysis, the high prevalence of LGLs in CB observed by us may be similar to the previously reported high frequency of NK cells in CB [13]. The CB-derived $\mathrm{NK}$ cells may require further stimulation in order to achieve maturation and functionality of PB-derived NK cells [20].

Generally, the immune responses of neonates are considered to be less effective than those of mature immune systems [21]. In this context, it is interesting to note the low presence of basophils (an average of $0.75 \%$ ) in the CB samples, and the near absence of these cells from preterm CB samples. Basophils participate in the regulation of type 2 immune responses by the production of cytokines, such as IL-4, IL-6, and TNFa [22, 23], and their low levels in the $\mathrm{CB}$ may be associated with the immature state of the immune system.

Normal Values and Prevalence of Aberrant Leukocytes in Cord Blood
We conclude that the data presented herein point to a unique maturation status of immune cells in the $\mathrm{CB}$, accompanied with exceptional morphological features, such as a high prevalence of LGLs and aberrant lymphocytes, which could be considered as abnormal in other age-groups. The differentials of preterm CB samples differ significantly from that of term CB samples, including the reverse of neutrophils/lymphocytes ratio, which may stem from different maturation status of the immune system, and alterations of hematopoiesis during gestation [17]. Morphological assessment of $\mathrm{CB}$ and newborn blood films is always a challenge, specifically for morphologists practicing adult blood film analysis. While aberrations in lymphoid cells morphology were not yet studied in CB samples, it was established that $\mathrm{CB}$ lymphocytes are significantly different compared to adult lymphocytes with respect to signaling, cytokines, and functional responses [20, 24, 25]. Hence, cells that appear abnormal in CB samples may, in fact, represent a normal, less mature stage of the immune system development, and not a source of concern.

\section{Acknowledgments}

We acknowledge Esther Eshkol, MA who provided assistance in preparing and editing the manuscript.

\section{Statement of Ethics}

The research was conducted ethically in accordance with the World Medical Association Declaration of Helsinki and by the local Institutional Review Board (IRB) at Tel Aviv Medical Center (Protocol No. 0501-20-TLV). Written informed consent was exempted by the IRB.

\section{Conflict of Interest Statement}

The authors have no conflicts of interest to declare.

\section{Funding Sources}

There were no funding sources for this work. 


\section{Author Contributions}

B.K. designed and performed the study, and wrote the first draft. D.B., S.K., J.H., and C.R. made substantial contributions to the data analysis and interpretation of the work. R.M. made substantial contributions to the conception, design, and data interpretation, supervised the study, and revised the manuscript for important intellectual content. All authors revised the draft and had final approval of the version to be submitted. Lastly, all of the authors agreed to be accountable for all aspects of the work ensuring that questions related to the accuracy and integrity of any part of the work are appropriately investigated and resolved.

\section{Data Availability Statement}

All raw data included in this manuscript are available from the corresponding author upon request.

\section{References}

1 Diez B, Genovese P, Roman-Rodriguez FJ, Alvarez L, Schiroli G, Ugalde L, et al. Therapeutic gene editing in CD34 hematopoietic progenitors from Fanconi anemia patients. EMBO Mol Med. 2017;9:1574-88.

2 Singh AK, McGuirk JP. Allogeneic stem cell transplantation: a historical and scientific overview. Cancer Res. 2016;76:6445-51.

3 Medhekar SK, Shende VS, Chincholkar AB. Recent stem cell advances: cord blood and induced pluripotent stem cell for cardiac regeneration: a review. Int J Stem Cells. 2016;9(9): 21-30.

4 Peeters B, Geerts I, Van Mullem M, Micalessi I, Saegeman V, Moerman J. Post-test probability for neonatal hyperbilirubinemia based on umbilical cord blood bilirubin, direct antiglobulin test, and $\mathrm{ABO}$ compatibility results. Eur J Pediatr. 2016;175:651-7.

5 Cizmeci MN, Kara S, Kanburoglu MK, Simavli S, Duvan CI, Tatli MM. Detection of cord blood hepcidin levels as a biomarker for earlyonset neonatal sepsis. Med Hypotheses. 2014; $82: 310-2$.

6 Su H, Chang SS, Han CM, Wu KY, Li MC, Huang CY, et al. Inflammatory markers in cord blood or maternal serum for early detection of neonatal sepsis-a systemic review and meta-analysis. J Perinatol. 2014;34:268-74.

7 Prakash N, Decristofaro J, Maduekwe ET. One less painful procedure: using umbilical cord blood as alternative source to admission complete blood count. Am J Perinatol. 2017; 34:1178-84.

8 Greer R, Safarulla A, Koeppel R, Aslam M, Bany-Mohammed FM. Can fetal umbilical venous blood be a reliable source for admission complete blood count and culture in NICU patients? Neonatology. 2019;115:4958.
9 Baer VL, Lambert DK, Carroll PD, Gerday E, Christensen RD. Using umbilical cord blood for the initial blood tests of VLBW neonates results in higher hemoglobin and fewer RBC transfusions. J Perinatol. 2013;33:363-5.

10 Glasser L, Sutton N, Schmeling M, Machan JT. A comprehensive study of umbilical cord blood cell developmental changes and reference ranges by gestation, gender and mode of delivery. J Perinatol. 2015;35:469-75.

11 Gac F, Thibert JB, Le Berre C, Le Priol J, Semana G, Fest T, et al. Evaluation of CytoDiff ${ }^{\mathrm{m}}$ on cord blood WBC differential. Int $J$ Lab Hematol. 2013;35:46-54.

12 Roberts I, O’Connor D, Roy A, Cowan G, Vyas P. The impact of trisomy 21 on foetal haematopoiesis. Blood Cells Mol Dis. 2013; 51:277-81

13 Sarvaria A, Jawdat D, Madrigal JA, Saudemont A. Umbilical cord blood natural killer cells, their characteristics, and potential clinical applications. Front Immunol. 2017;8:3296.

14 Palmer L, Briggs C, McFadden S, Zini G, Burthem J, Rozenberg G, et al. ICSH recommendations for the standardization of nomenclature and grading of peripheral blood cell morphological features. Int J Lab Hematol. 2015; 37:287-303.

15 Bain BJ. Diagnosis from the blood Smear. N Engl J Med. 2005;353(5):498-507.

16 Roberts I, Alford K, Hall G, Juban G, Richmond $\mathrm{H}$, Norton $\mathrm{A}$, et al. GATA1-mutant clones are frequent and often unsuspected in babies with down syndrome: identification of a population at risk of leukemia. Blood. 2013; 122:3908-17.

17 Rolim ACB, Lambert MA, Borges JPG, Abbas SA, Bordin JO, Langhi Junior DM, et al. Blood cells profile in umbilical cord of late preterm and term newborns. Rev Paul Pediatr. 2019; $37: 264-74$.
18 Narayanan S. The preanalytic phase. An important component of laboratory medicine. Am J Clin Pathol. 2000;113:429-52.

19 Vives-Corrons JL, Briggs C, Simon-Lopez R, Albarede S, de la Salle B, Flegar-Meatrii Z, et al. Effect of EDTA-anticoagulated whole blood storage on cell morphology examination. A need for standardization. Int J Lab Hematol. 2014;36:222-6.

20 Shereck E, Day NS, Awasthi A, Ayello J, Chu $\mathrm{Y}, \mathrm{McGuinn} \mathrm{C}$, et al. Immunophenotypic, cytotoxic, proteomic and genomic characterization of human cord blood vs. peripheral blood CD56Dim NK cells. Innate Immun. 2019;25: 294-304.

21 Adkins B, Leclerc C, Marshall-Clarke S. Neonatal adaptive immunity comes of age. Nat Rev Immunol. 2004;4:553-64.

22 Wynn TA. Type 2 cytokines: mechanisms and therapeutic strategies. Nat Rev Immunol. 2015;15(5):271-82.

23 Yuk CM, Park HJ, Kwon BI, Lah SJ, Chang J, Kim JY, et al. Basophil-derived IL-6 regulates $\mathrm{T}(\mathrm{H}) 17$ cell differentiation and CD4 $\mathrm{T}$ cell immunity. Sci Rep. 2017;7:417-44.

24 Nupponen I, Kuuliala A, Siitonen S, Repo H, Kuuliala K. Cord blood monocytes, neutrophils and lymphocytes from preterm and fullterm neonates show multiple aberrations in signalling profiles measured using phosphospecific whole-blood flow cytometry. Scand J Immunol. 2013;78:426-38.

25 Sato K, Nagayama H, Takahashi TA. Aberrant CD3- and CD28-mediated signaling events in cord blood $\mathrm{T}$ cells are associated with dysfunctional regulation of Fas ligandmediated cytotoxicity. J Immunol. 1999;162: 4464-71. 